\title{
AVALIAÇÃO DE ÍNDICES ESPECTRAIS OBTIDOS COM IMAGENS SENTINEL 2 E LANDSAT 8 ANTES E APÓS ROMPIMENTO DA BARRAGEM DA MINA DO FEIJÃO, BRUMADINHO - MG
}

\author{
EVALUATION OF SPECTRAL INDEXES OBTAINED WITH SENTINEL 2 AND LANDSAT 8 \\ IMAGES BEFORE AND AFTER BREAK OF THE FEIJÃO MINE DAM, BRUMADINHO - MG
}

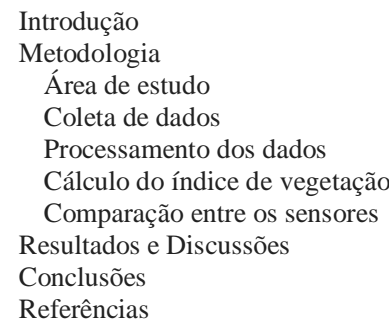

Débora Joana DUTRA, Marcos Antônio Timbó ELMIRO

Universidade Federal de Minas Gerais. Avenida Presidente Antônio Carlos, 6627 - Pampulha, Belo Horizonte - MG. E-mails: ddutra.ambiental@gmail.com; timboelmiro@gmail.com

\begin{abstract}
RESUMO - Métodos e dados de sensoriamento remoto têm sido largamente utilizados em diversas aplicações de análises ambientais. Dentro desse aspecto, a sua utilização tem auxiliado de forma efetiva em análises de mudanças do solo, bem como, nos estudos de vegetação. Os índices de vegetação permitem realizar análises e estudos da biomassa e do vigor vegetativo apresentado por uma região de forma simples e eficaz. Esse artigo realizou uma análise do Índice de Vegetação de Diferença Normalizada (NDVI), comparando os resultados obtidos a partir de imagens dos sensores Operational Land Imager (OLI) e Multispectral Instrument (MSI). Além disso, o estudo realizou uma comparação entre a aplicação do índice em imagens com e sem a utilização de técnicas de correção da atmosfera. A área de estudo é a região da mina do feijão, em Brumadinho - MG, onde ocorreu o rompimento da barragem de rejeitos em 25 de janeiro de 2019. Os resultados encontrados permitiram realizar uma comparação entre as imagens, com e sem a utilização de técnicas de pré-processamento para correção atmosférica, e analisar o comportamento apresentado pela região antes e depois do acidente.

Palavras-chave: Sensoriamento Remoto; NDVI, atmosfera.
\end{abstract}

\begin{abstract}
Remote sensing techniques have been largely used in several environmental analyzes. So they have effectively helped many studies related to soil changes and vegetation health situation. The vegetation index allows straightforward effective analysis of the biomass and the vegetative vigor presented by a region. This paper performed an analysis of the Normalized Difference Vegetation Index (NDVI), by comparing the results obtained with Operational Land Imager (OLI) and Multispectral Instrument (MSI) sensor images. Furthermore, it compared the application of index in images with and without the atmospheric correction techniques. The study area consisted in the region of the Feijão Mine, in Brumadinho - MG where a dam disruption occurred on January 25, 2019. The study results allowed comparing the images with and without the use atmospheric correction as well as analyzing the region behavior before and after the accident.
\end{abstract}

Keywords: Remote Sensing; NDVI, Atmosphere.

\section{INTRODUÇÃO}

Algoritmos e métodos de processamento digital de imagens (PDI) aplicados aos dados de sensoriamento remoto, de crescente disponibilidade e rapidez de acesso, têm possibilitado cada vez mais as análises de diversos ambientes e recursos da Terra, ampliando significativamente as possibilidades de novos estudos nas áreas de meio ambiente $\mathrm{e}$ de ciências da terra. Entretanto, para aplicações adequadas das técnicas de PDI é de grande importância a diminuição ou eliminação de ruídos presentes nas imagens inerentes ao próprio processo de imageamento.

Os efeitos da atmosfera podem ocasionar alterações no brilho da imagem e a redução do contraste entre os alvos, comprometendo a interpretação e os demais processos de extração de informações. Tais efeitos resultam diretamente dos fenômenos da absorção e espalhamento da radiação pelos componentes da atmosfera, podendo ocasionar graves erros na imagem. Visando diminuir os efeitos indesejáveis ocasionados pelos constituintes da atmosfera, diversos modelos de correção têm sido criados para atingir melhores resultados no processo de análise das imagens (Maranhão et al., 2017).

Devido à facilidade de acesso, as imagens dos satélites Landsat 8 e Sentinel 2 têm sido bastante utilizadas para aplicação de técnicas de PDI 
relacionadas a cálculos de índices de vegetação em muitos estudos de diferentes áreas da ciência. Entretanto, para atingir resultados precisos a utilização dessas imagens requer a aplicação de técnicas de pré-processamento adequadas para a atenuação dos ruídos.

As principais bandas utilizadas no cálculo dos índices de vegetação são referentes às regiões do espectro visível e do infravermelho próximo. Um exemplo muito difundido de aplicação é o índice de vegetação por diferença normalizada (NDVI), criado por Rouse et al. (1973, 1974). O NDVI varia entre - 1 e 1 e permite o estudo da vegetação de uma região de forma bastante rápida e direta. De modo geral, quanto maior o valor do índice, maior o vigor vegetativo apresentado por uma região.

A frequente utilização desses índices se deve ao fato de serem capazes de auxiliar no estabelecimento de soluções, bem como inferir sobre as modificações das mudanças da paisagem. Além disso, possibilitam as análises sobre a dinâmica da vegetação, análises de áreas degradadas ou processos em áreas que sofreram impactos ambientais (Da Silva \& Almeida; 2016).

Assim, dado o massivo uso dos índices, as diferentes fontes de dados disponíveis para seus equacionamentos e a questão dos tratamentos de ruídos nas fontes de dados para garantia da qualidade dos resultados o presente estudo analisa, à luz dessas questões, diferenças apresentadas no cálculo de índices de vegetação antes e depois do rompimento da barragem da Mina do Feijão usando imagens dos satélites Lansdat 8 (sensor OLI) e Sentinel 2 (sensor MSI). O estudo avalia o efeito da correção atmosférica nos resultados dos índices.

\section{METODOLOGIA}

\section{Área de estudo}

As análises incorporadas na metodologia do estudo foram realizadas na região do rompimento da barragem de rejeitos da Mina do Feijão, em Brumadinho - MG, conforme figura 1. Em 25 de janeiro de 2019 a região sofreu um grande impacto ambiental oriundo do rompimento da barragem de rejeitos explorada pela mineradora VALE SA. O acidente foi responsável por contaminar grande parte dos corpos d'água da região chegando ao rio principal da bacia, o Paraopeba.

\section{Coleta de dados}

Foram utilizadas as bandas do espectro vermelho e infravermelho próximo provenientes dos satélites Landsat 8 (sensor OLI) e Sentinel 2 (sensor MSI), que correspondem às especificações para os cálculos do índice de vegetação utilizado (NDVI). .

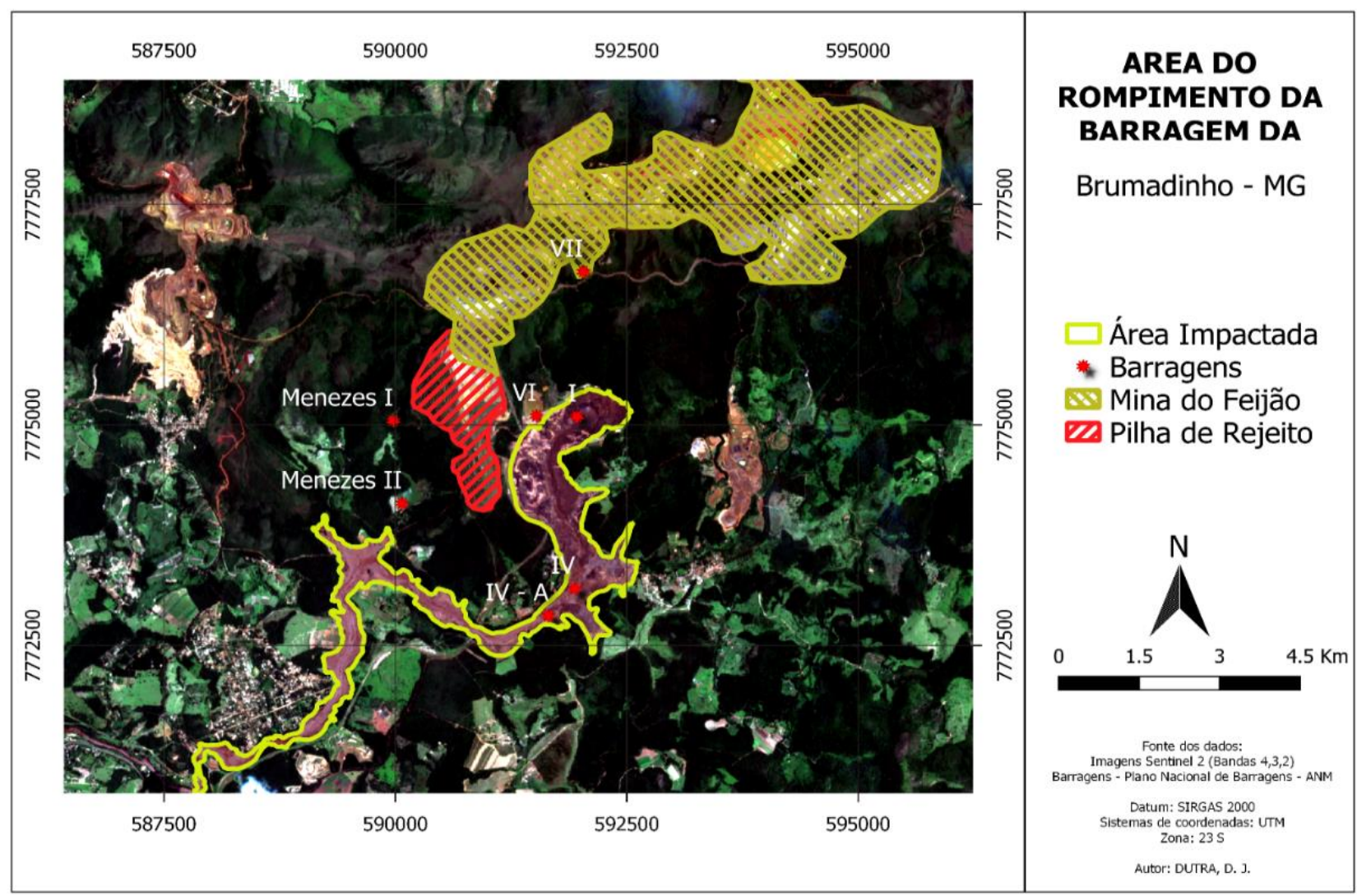

Figura 1 - Área de estudo, região da barragem da mina do Feijão em Brumadinho, MG. 
Adquiriu-se uma cena de cada satélite no período anterior e posterior à data de rompimento (25 de janeiro de 2019), conforme apresentado na tabela 1 .

Tabela 1 - Dados referentes às imagens Sentinel 2 e Landsat 8.

\begin{tabular}{c|c|c}
\hline Data & Sensor & Situação \\
\hline $14 / 01 / 2019$ & OLI & Antes do acidente \\
\hline $22 / 01 / 2019$ & MSI & Antes do acidente \\
\hline $30 / 01 / 2019$ & OLI & Depois do acidente \\
\hline $01 / 02 / 2019$ & MSI & Depois do acidente \\
\hline
\end{tabular}

Devido às divergências nas características de resolução temporal de cada satélite, não foi possível obter uma imagem do mesmo dia para os dois sensores, tendo sido buscadas as datas mais próximas possíveis para manter ao máximo as características e similaridades das regiões. As imagens foram escolhidas com uma porcentagem de nuvens de até $10 \%$, visando minimizar interferência de ruídos.

\section{Processamento dos dados}

O primeiro passo do trabalho consistiu na montagem dos cenários, visando avaliar o comportamento dos índices diante dos efeitos atmosféricos. Assim, optou-se pela abordagem das duas situações a seguir:

- Cenário 1: cálculo dos índices de vegetação a partir das imagens sem o pré-processamento de correção atmosférica; e

- Cenário 2: cálculo dos índices de vegetação a partir das imagens com o pré-processamento da correção atmosférica. Neste caso, ambas as imagens foram submetidas ao mesmo algoritmo de correção. O processo foi realizado da mesma forma visando minimizar possíveis discrepâncias entre os dados.

Os dados foram processados no ambiente QGIS (2018), versão 3.4.6. A correção atmosférica para o Cenário 2 foi realizada por meio do algoritmo incorporado no semi-automatic classification plugin (SCP).

O SCP consiste em uma ferramenta de classificação supervisionada que permite o préprocessamento bem como o pós-processamento para imagens de alguns satélites, como o Sentinel 2 e Landsat 8. Os dados de Níveis Digitais foram convertidos em valores de reflectância de superfície no processamento de correção atmosférica.

Devido às resoluções espaciais diferentes apresentadas pelo satélite Sentinel $2(10 \mathrm{~m})$ e Landsat $8(30 \mathrm{~m})$, foi necessário realizar a equalização das resoluções.

Na compatibilização de diferentes resoluções é recomendável que o processo de reamostragem converta os pixels para a resolução espacial mais grosseira utilizada na pesquisa. Dessa forma, a resolução das imagens MSI $(10 \mathrm{~m})$ foi degradada para um tamanho de pixel de $30 \mathrm{~m}$.

\section{Cálculo do índice de vegetação}

A tabela 2 apresenta detalhes das bandas dos sensores OLI e MSI utilizadas para os cálculos dos índices de vegetação.

Tabela 2 - Bandas espectrais consideradas no estudo.

\begin{tabular}{c|c|c}
\hline OLI - Landsat 8 & Nomenclatura espectral da banda & MSI - Sentinel 2 \\
\hline B04 $(0,640-0,690 \mu m)$ & Vermelho & B04 $(0,650-0,680 \mu m)$ \\
\hline B05 $(0,850-0,880 \mu m)$ & Infravermelho próximo & $\mathrm{B} 08(0,698-0,713 \mu m)$ \\
\hline
\end{tabular}

$O$ índice de vegetação por diferença normalizada (NDVI) escolhido para uso neste estudo é definido pela Equação 1, proposta por Rouse et al. (1973, 1974).

Sua variação oscila de -1 a 1 , de forma que quanto maior o valor calculado maior o vigor vegetativo apresentado pela região.

Onde

$$
\mathbf{N D V I}=\frac{\mathbf{I V P}-\mathbf{V}}{\mathbf{I V P}+\mathbf{V}} \quad \text { Eq. (1) }
$$

NDVI = Índice de vegetação por diferença normalizada

$\mathrm{IVP}=$ Banda do infravermelho próximo $(\mathrm{OLI}=$ 5 e MSI $=8$ )

$\mathrm{V}=$ Banda do vermelho $(\mathrm{OLI}=4$ e MSI $=4)$

\section{Comparação entre os sensores}

A comparação entre os índices obtidos a partir dos dados dos dois sensores foi realizada mediante o ajuste dos dados por meio de um modelo de uma regressão linear e do cálculo do coeficiente de correlação de Pearson, representado pela Equação 2.

$$
r=\frac{\sum_{i=1}^{n}\left(x_{i}-\bar{x}\right)\left(y_{i}-\bar{y}\right)}{\sqrt{\sum_{i=1}^{n}\left(x_{i}-\bar{x}\right)^{2} \sum_{i=1}^{n}\left(y_{i}-\bar{y}\right)^{2}}} \quad \text { Eq. (2) }
$$

Onde

$\mathrm{r}=$ coeficiente de Pearson

$x_{i}=$ valores de um conjunto de medidas

$\bar{x}=$ média dos valores de um conjunto de medidas 
$y_{i}=$ valores de um conjunto de medidas correspondentes $\bar{y}=$ média dos valores de um conjunto de medidas correspondentes

\section{RESULTADOS E DISCUSSÕES}

Os resultados do trabalho indicaram que a região de estudo sofreu grande impacto após o rompimento da barragem da mina do feijão, conforme análises extraídas dos dois cenários analisados. Borges et al. (2010), em seu estudo sobre vegetação no estado de Minas Gerais identificou que regiões com valores de NDVI abaixo de zero correspondem às áreas com ausência de vegetação e que regiões com valores maiores que 0,6 correspondem às áreas com vigor vegetativo muito alto. Relações similares foram corroboradas pelos estudos de Aires et al. (2017) na região da bacia do rio Paraopeba.

Observou-se um crescimento das regiões que apresentaram valores de NDVI acima de 0 após o cenário de rompimento da barragem. Além disso, foi possível identificar uma grande variação entre os resultados encontrados nos cenários. O Cenário 2 apresentou, para ambos sensores, uma identificação do vigor vegetativo da região maior que o cenário 1 , principalmente nos resultados oriundos do satélite Landsat 8, figura 2. Ficou claro, na avaliação dos cenários, que a atmosfera pode exercer influência nos resultados de índice de vegetação obtidos. De acordo com Dos Santos et al. (2017), devido às imagens serem obtidas a grandes distâncias da superfície a atmosfera pode influenciar na radiação que atinge o sensor, sendo um dos causadores de degradação das imagens, o que compromete a análise e interpretação dos dados oriundos de sensores. A influência da atmosfera depende do comprimento de onda da radiação captada pelo sensor. Dessa forma, essa interferência pode variar de banda para banda.

A aplicação de técnicas de correção atmosférica em imagens de satélites possibilita a extração de informações mais fidedignas do terreno, conduzindo a uma melhor caracterização das paisagens e a tomadas de decisões relativas ao uso e conservação de recursos naturais mais acertadas (Magalhães et al., 2017).

As análises a partir do NDVI possibilitaram uma melhor visualização e entendimento mais consistente da dinâmica a qual foi submetida à região, bem como permitiram identificar um aumento dos valores estatísticos gerais do índice após a aplicação do algoritmo de correção atmosférica SCP, conforme resultados apresentados na tabela 3. A análise dos resultados possibilitou inferir que a aplicação do SCP produziu resposta de maior vigor da vegetação se comparado com a mesma análise sem a correção atmosférica.

Tabela 3 - Variação básica estatística das análises realizadas em cada cenário de estudo.

\begin{tabular}{c|c|c|c|c|c|c}
\hline \multirow{2}{*}{ Cenário } & Satélite & Datas & Min & Max & Média & \multirow{2}{*}{ Desv. Padrão } \\
\hline \multirow{3}{*}{1} & \multirow{3}{*}{ Landsat 8 } & $14 / 01 / 2019$ & $-0,269$ & 0,639 & 0,369 & 0,124 \\
\cline { 3 - 7 } & \multirow{2}{*}{ Sentinel 2 } & $30 / 01 / 2019$ & $-0,280$ & 0,618 & 0,354 & 0,127 \\
\cline { 3 - 7 } & & $22 / 01 / 2019$ & $-0,372$ & 0,837 & 0,564 & 0,203 \\
\hline \multirow{3}{*}{2} & \multirow{2}{*}{ Landsat 8 } & $14 / 01 / 2019$ & $-0,653$ & 0,864 & 0,621 & 0,198 \\
\cline { 3 - 7 } & \multirow{2}{*}{ Sentinel 2 } & $30 / 01 / 2019$ & $-0,728$ & 0,844 & 0,605 & 0,198 \\
\cline { 3 - 7 } & & $22 / 01 / 2019$ & $-0,349$ & 0,835 & 0,564 & 0,202 \\
\hline
\end{tabular}

Após o rompimento da barragem, nos dois cenários, ocorreu um aumento dos valores mínimos de NDVI derivados dos sensores, bem como uma diminuição dos valores máximos. Isso pode ser explicado pelo fato de parte da área ter sido inundada pela lama, ocasionando diminuição da resposta do índice na região decorrente da supressão de parte da vegetação após o rompimento da barragem. No geral a média de NDVI encontrada foi próxima de 0,6 , exceto para os resultados oriundos do satélite Landsat 8 antes da tragédia.

Observou-se que os dados de NDVI no cenário 1, tanto antes quanto depois do rompimento da barragem, apresentaram valores entre 0,6 e 1 para o satélite Sentinel 2 (MSI) e entre 0,4 e 0,6 para os dados do satélite Landsat 8 (OLI).. 

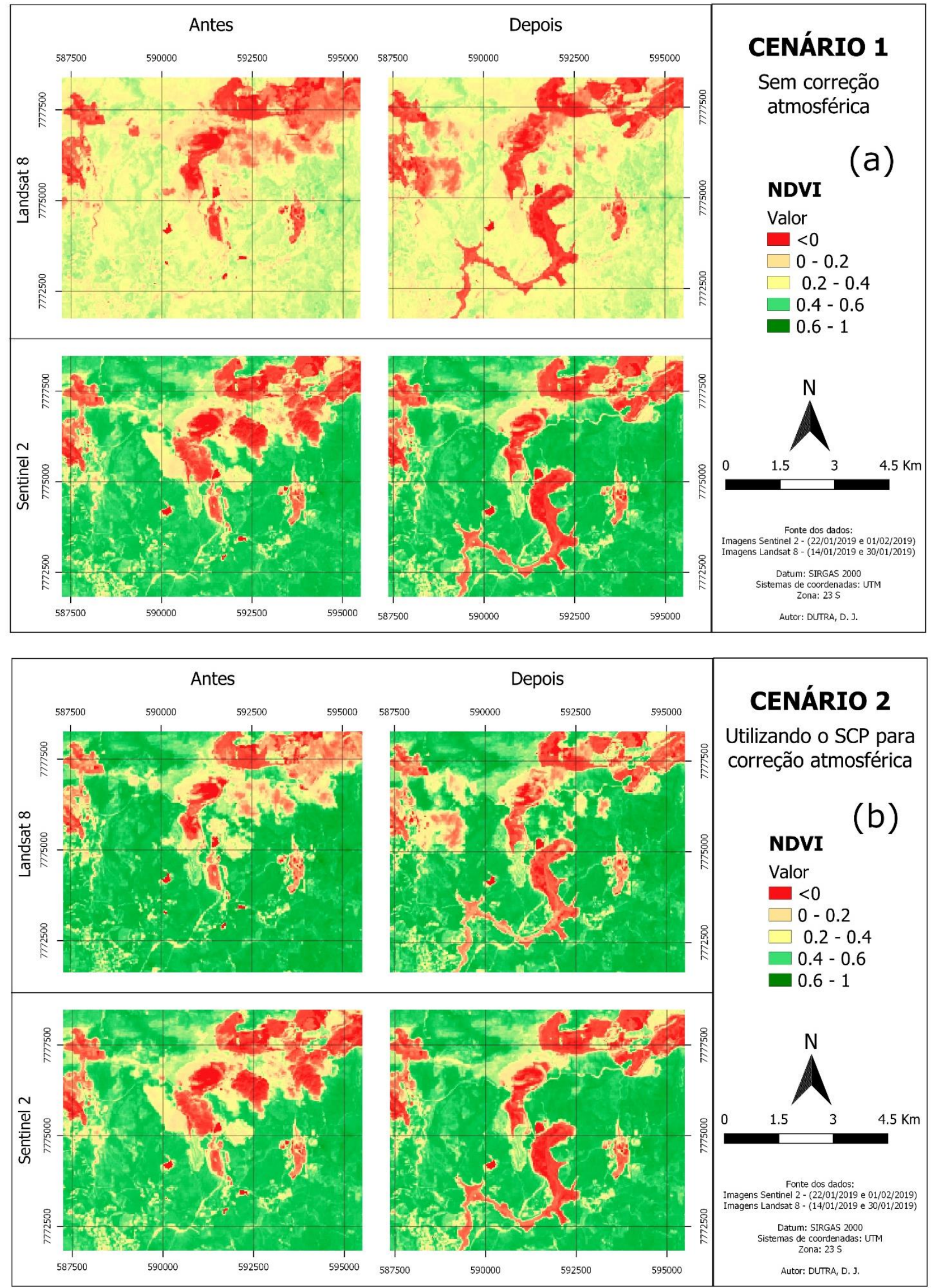

Figura 2 - NDVI apresentado pela região antes e depois do acidente (comparação entre os dados obtidos dos satélites Landsat 8 e Sentinel 2), (a) Cenário 1 e (b) Cenário 2

No cenário 2, para ambos sensores, os dados se concentraram na faixa de 0,6 a 1 , conforme figura 3.

A associação entre os dados de NDVI obtidos 
a partir dos sensores apresentaram uma correlação positiva tanto antes como depois do acidente, figura 4.

Foi possível observar, nos dados do cenário 1, uma maior concentração de valores positivos, se comparado com os dados apresentados pelo
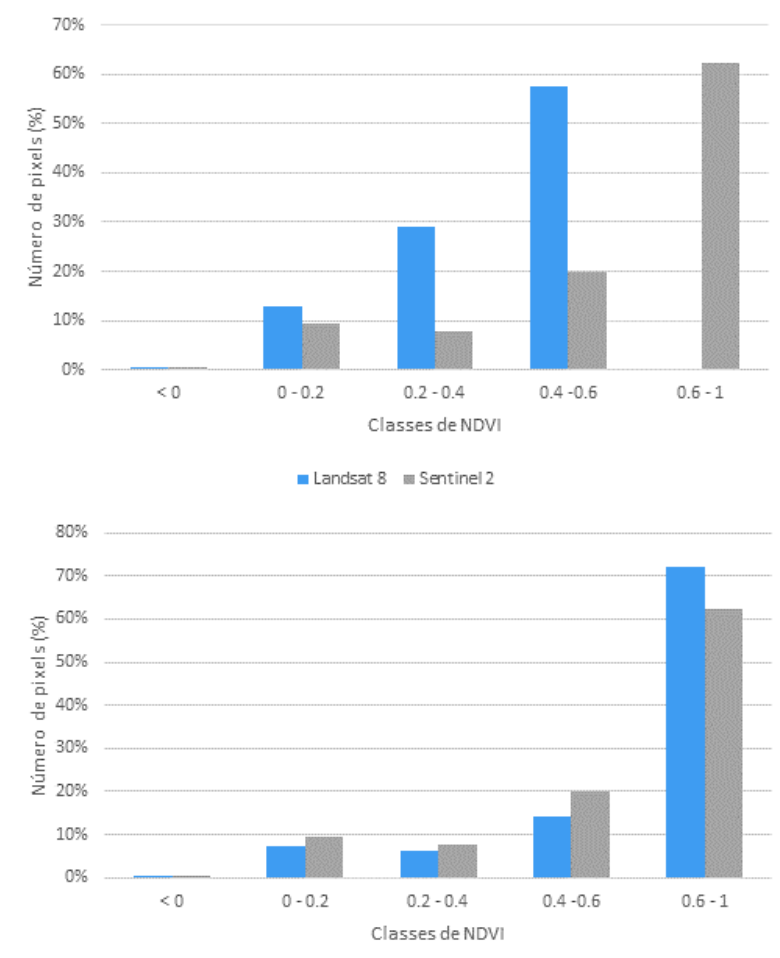

(a) cenário 2.

Figueiredo Filho et al. (2014), relata que nesse tipo de correlação quando um dos eixos apresenta um aumento o outro também aumenta. Dessa forma, quanto maiores os valores de $\mathrm{x}$ maiores serão os valores de associado.
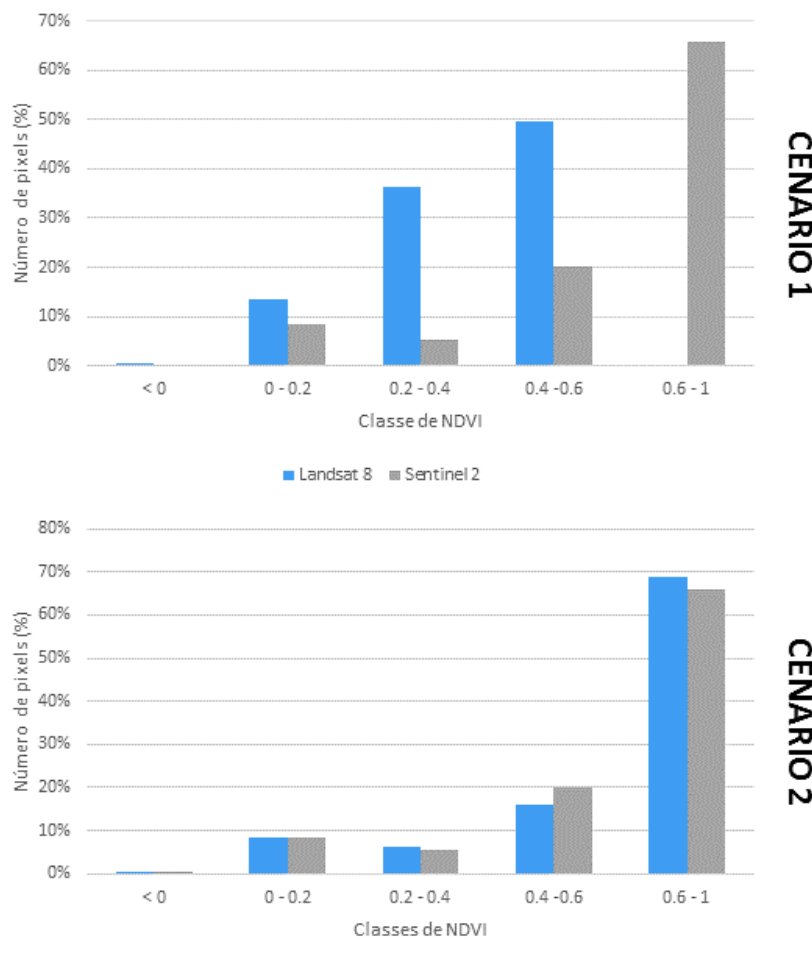

(b)

Figura 3 - Variação dos dados do NDVI: (a) antes e (b) após a ruptura da barragem da Mina do feijão nos cenários 1 e 2.

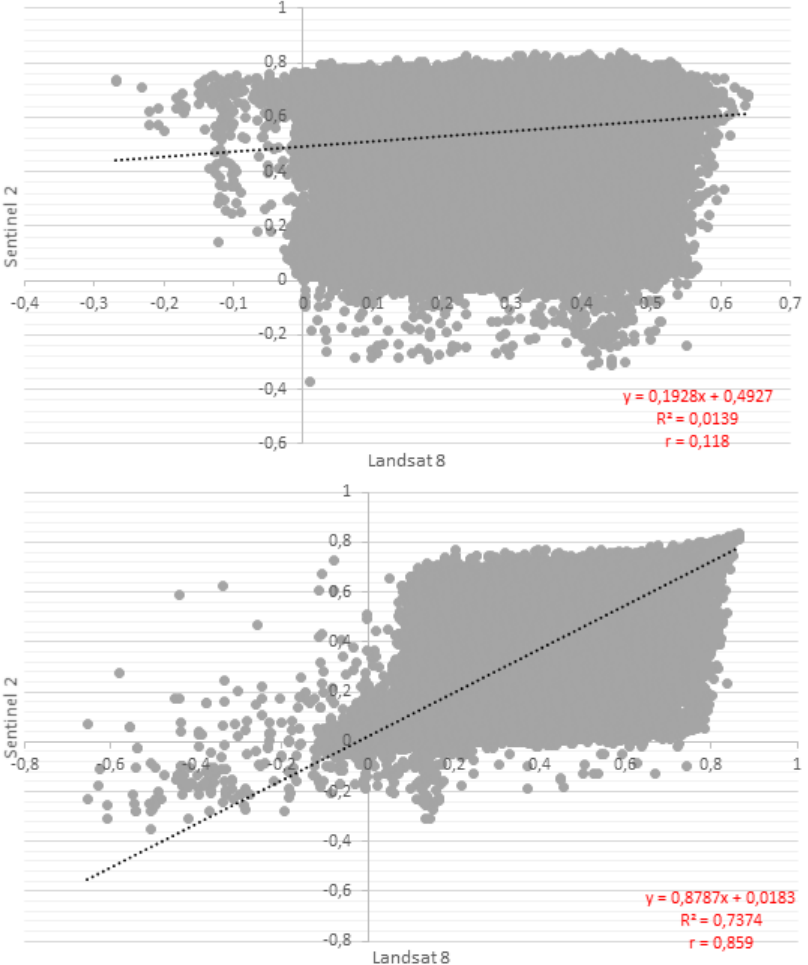

(a)

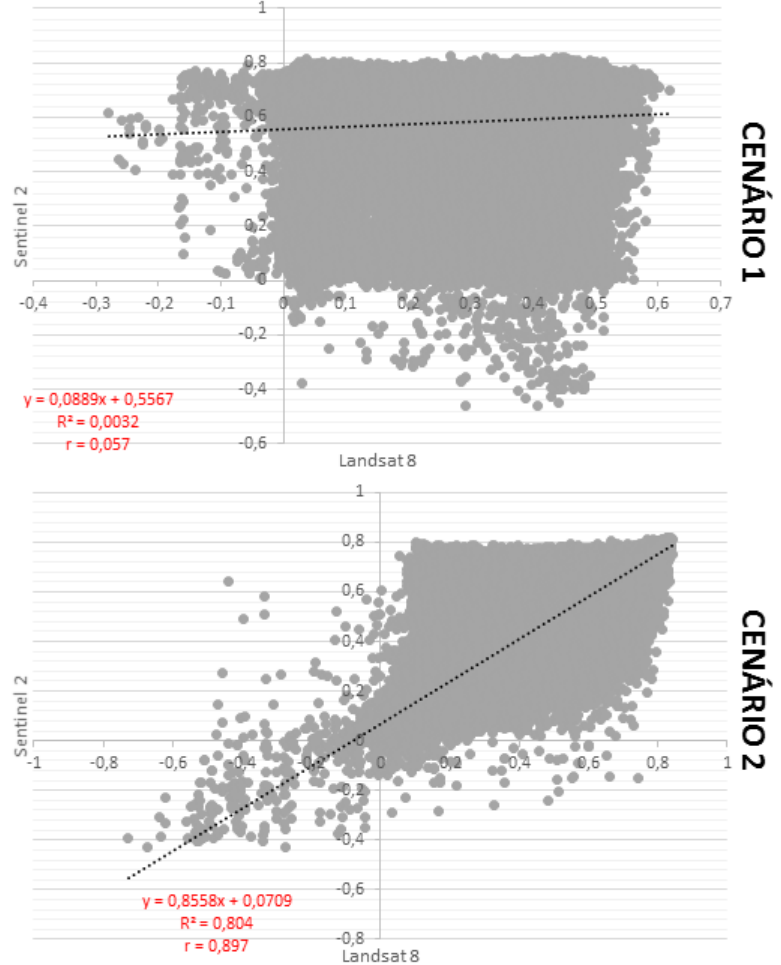

(b)

Figura 4 - Correlação entre os dados do NDVI dos sensores: (a) antes e (b) após a ruptura da Mina d0 Feijão nos cenários 1 e 2 . 
A tabela 4 apresenta os valores do coeficiente de Pearson apresentado em cada análise de cenário. Foi possível identificar que os dados que receberam tratamento para correção atmosférica apresentaram uma correlação mais forte, se comparado com os dados sem préprocessamento, próximo a 0,9 . De acordo com Martins (2014), quanto maior o valor absoluto do coeficiente de correlação de Pearson, maior o grau de associação entre os dados em uma regressão linear, ou seja, quanto mais próximo de 1, maior forte é a associação linear entre os dados. Dessa forma, é possível identificar que os dados dos satélites Landsat 8 e Sentinel 2 se correlacionaram melhor após a aplicação de técnicas de correção atmosférica.

Tabela 4 - Comparação entre os índices espectrais calculados nos cenários estabelecidos.

\begin{tabular}{c|c|c}
\hline & Processamento & r \\
\hline \multirow{2}{*}{$\begin{array}{c}\text { Antes do } \\
\text { acidente }\end{array}$} & Sem correção & 0,057 \\
\cline { 2 - 3 } & Com correção & 0,859 \\
\hline \multirow{2}{*}{$\begin{array}{c}\text { Depois do } \\
\text { acidente }\end{array}$} & Sem correção & 0,127 \\
\cline { 2 - 3 } & Com correção & 0,897 \\
\hline
\end{tabular}

\section{CONCLUSÃO}

Os resultados demonstraram que após o rompimento da barragem de rejeitos ocorreu um aumento dos valores mínimos de NDVI e a diminuição dos valores máximos. O principal fator associado explicativo dessa mudança é o fato de parte da vegetação da área ter sido suprimida pelo rejeito da barragem.

Identificou-se uma mudança das classes de NDVI entre o período anterior e posterior ao rompimento da barragem da Mina do Feijão. No cenário 1, tanto antes quanto depois do rompimento da barragem, o NDVI apresentou valores entre 0,6 e 1 para o satélite Sentinel 2 (MSI) e entre 0,4 e 0,6 para os dados do satélite Landsat 8 (OLI). No cenário 2, para ambos sensores, os dados se concentraram na faixa de 0,6 a 1 . No processo de análise de cada cenário foi possível verificar a interferência da atmosfera de forma significativa no cálculo do NDVI. Além disso, foi possível identificar que a aplicação do procedimento de correção atmosférica das imagens (cenário 2) aumentou o grau de correlação entre os resultados de NDVI obtidos a partir dos sensores OLI e MSI.

\section{REFERENCIAS}

AIRES, U.R.V.; NETO, J.O.M.; CAMPOS, J.A. Uso de NDVI para análise do comportamento do regime de vazões do rio Paraopeba, MG. Scientia Agraria, v. 18, n. 3, p. 8-19, 2017.

BORGES, L.A. Estimativa de NDVI no Estado de Minas Gerais utilizando imagens dos satélites CERBS_2BCD e MSGISEVIRI. Lavras. 60 p. 2010. Dissertação (Mestrado em Engenharia Agrícola) - Universidade Federal de Lavras.

DA SILVA, G.J.F. \& ALMEIDA, N.V. Degradação ambiental no município de Parari-PB: uma análise por meio de sensoriamento remoto. Revista Geografar, v. 10, n. 2, p. 140164, 2016.

DOS SANTOS, T.C.C.; ANTUNES, M.A.H.; SEOANE, J.C.S.; SOUSA, G.M. Comparação de modelos de correção atmosférica para imagem WorldView-2. Revista Brasileira de Cartografia, v. 69, n. 2, p. 229-240, 2017

FIGUEIREDO FILHO, D.B.; ROCHA, E.C.; SILVA JÚNIOR, J.A.; RANULFO, P.; NEVES, J.A.B.; SILVA, M.B. Desvendando os mistérios do coeficiente de correlação de Pearson: o retorno. Leviathan, São Paulo, n. 8, p. 66-95, 2014 MARANHÃO, D.D.C.; PEREIRA, M.G.; COSTA, E.M.; ANJOS, L.H.C. Correção de Imagens e caracterização do uso da terra no município de Pinheiral, Estado do Rio de Janeiro, Brasil. Caminhos de Geografia, v. 18, n. 62, p. 174-188, 2017.
MARTINS, M.E.G. Coeficiente de correlação amostral. Revista de Ciência Elementar, v. 2, n. 2, 4 p., 2014.

QGIS, 2018. QGIS Geographic Information System. Open Source Geospatial Foundation Project.

ROUSE, J.W; HAAS, R. H.; SCHELL, J.A.; DEERING, D.W; HARLAN, J. C. Monitoring the venal advancement and retro gradation (green wave effect) of natural vegetation. NASA/GSFC Type III Final Report, Greenbelt, 1974, 371 p. ROUSE, J.W; HAAS, R.H.; SCHELL, J.A.; DEERING, D.W. Monitoring vegetation systems in the Great Plains with ERTS. Proceedings of the third erts symposium, Washington, p. 309-317, p. 309, 1973.

Submetido em 5 de setembro de 2019 Aceito para publicação em 20 de maio de 2020 